\title{
ON ONE-DIMENSIONAL LEIBNIZ CENTRAL EXTENSIONS OF A FILIFORM LIE ALGEBRA
}

\author{
ISAMIDDIN S. RAKHIMOV ${ }^{凶}$ and MUNTHER A. HASSAN
}

(Received 25 April 2010)

\begin{abstract}
The paper deals with the classification of Leibniz central extensions of a filiform Lie algebra. We choose a basis with respect to which the multiplication table has a simple form. In low-dimensional cases isomorphism classes of the central extensions are given. In the case of parametric families of orbits, invariant functions (orbit functions) are provided.
\end{abstract}

2010 Mathematics subject classification: primary 17A32; secondary 17A60, 17B30, 13 A50.

Keywords and phrases: Lie algebra, filiform Leibniz algebra, isomorphism, invariant.

\section{Introduction}

Leibniz algebras were introduced by Loday [4]. A Leibniz algebra is a generalization of a Lie algebra: a skew-symmetric Leibniz algebra is a Lie algebra. The initial motivation of Loday to introduce this class of algebras was the search for an 'obstruction' to the periodicity in algebraic $K$-theory. Besides this purely algebraic motivation, certain relationships with classical geometry, noncommutative geometry and physics have recently been discovered. Leibniz algebras appear to be related in a natural way to several topics such as differential geometry, homological algebra, classical algebraic topology, algebraic $K$-theory, loop spaces, noncommutative geometry, quantum physics and so on, as a generalization of the corresponding applications of Lie algebras to these topics.

In 1891, Umlauf [7] initiated the study of the simplest nontrivial class of Lie algebras. In his thesis he presented a list of Lie algebras of dimension less than ten admitting a so-called adapted basis. (Lie algebras with this property have been called filiform Lie algebras.) Now it is well known that up to isomorphism there is only one such an algebra; the others are just linear deformations of it [3]. This is the filiform Lie algebra with the composition law $[\cdot, \cdot]$ given by

$$
\mu_{n}:\left[e_{i}, e_{0}\right]=e_{i+1}, \quad 1 \leq i \leq n-2,
$$

with respect to the adapted basis $\left\{e_{0}, e_{1}, \ldots, e_{n-1}\right\}$.

The research was supported by the Grant 01-12-10-978FR MOHE, Malaysia.

(C) 2011 Australian Mathematical Publishing Association Inc. 0004-9727/2011 \$16.00 
The outline of the paper is as follows. Section 2 is a brief introduction to Leibniz algebras and central extensions. Section 3 describes the behaviour of parameters under the isomorphism action (adapted base change). Sections 3.1-3.5 contain the main results of the paper, consisting of complete lists of all one-dimensional Leibniz central extensions of $\mu_{n}$, where $n=4, \ldots, 8$. We distinguish the isomorphism classes and show that they exhaust all possible cases. For parametric family cases the corresponding invariant functions are provided. Since proofs from the fivedimensional cases can be carried over to higher-dimensional cases by minor changes, we have chosen to omit their proofs. All details of the omitted proofs are available from the authors.

\section{Preliminaries}

Let $K$ be an algebraically closed field of characteristic 0 . A Leibniz algebra $L$ over $K$ is a vector space equipped with a bilinear map $[\cdot, \cdot]: L \times L \rightarrow L$ satisfying the Leibniz identity

$$
[x,[y, z]]=[[x, y], z]-[[x, z], y]
$$

for all $x, y, z \in L$.

If, in addition, $[x, x]=0$, for all $x \in L$, the Leibniz identity is equivalent to the Jacobi identity. In particular, a Lie algebra is an example of a Leibniz algebra.

The centre of a Leibniz algebra $L$ is defined as $C(L)=\{x \in L \mid[x, L]=$ $[L, x]=0\}$.

Let $L$ and $\hat{L}$ be two Leibniz algebras over a field $K$. The Leibniz algebra $\hat{L}$ is said to be a one-dimensional central extension of $L$ if there is a Leibniz algebra exact sequence $0 \longrightarrow K c \longrightarrow \hat{L} \longrightarrow L \longrightarrow 0$, where $K c$ is one-dimensional trivial Leibniz algebra and the image of $K c$ is contained in the centre of $\hat{L}$.

A Leibniz 2-cocycle on a Leibniz algebra $L$ is a $K$-valued form $\theta$ satisfying the condition

$$
\theta(x,[y, z])=\theta([x, y], z)-\theta([x, z], y) \text { for all } x, y, z \in L .
$$

If a Leibniz 2-cocycle $\theta$ is also antisymmetric, then by definition, $\theta$ is a Lie 2-cocycle. As in the Lie algebra case, one-dimensional Leibniz central extensions of a Leibniz algebra $L$ are uniquely determined by a Leibniz 2-cocycle on $L$. If a Leibniz 2-cocycle $\theta$ is induced by a linear map $v: L \rightarrow L$ (that is, $\theta(x, y)=v([x, y]))$, then $\theta$ is said to be trivial (or a coboundary), while the corresponding one-dimensional Leibniz central extension is also a trivial extension; that is, it is isomorphic to the direct sum of $L$ and $K$. Two Leibniz 2-cocycles $\theta$ and $\vartheta$ define the same central extension if their difference $\theta-\vartheta$ is a coboundary.

Given a Leibniz 2-cocycle $\theta$ on $L$, one can construct a one-dimensional Leibniz central extension $L_{\theta}=L \oplus K c$ of $L$ in a canonical way as follows:

$$
[x+v c, y+w c]_{L_{\theta}}=[x, y]_{L}+\theta(x, y) c, \quad x, y \in L, v, w \in K,
$$

where $[\cdot, \cdot]_{L}$ is the bracket on $L$. Every one-dimensional Leibniz central extension of $L$ can be obtained in this way. The following result is known. 
PROPOSITION 2.1 [5]. There exists a one-to-one correspondence between the set of equivalent classes of one-dimensional Leibniz central extensions of $L$ by $K$ and the second Leibniz cohomology group $H L^{2}(L, K)$.

In this paper we focus on one-dimensional Leibniz central extensions of the filiform Lie algebra $\mu_{n}$ denoted here by $C E\left(\mu_{n}\right)$.

Let $L$ be a Leibniz algebra. Define

$$
L^{1}=L, \quad L^{k+1}=\left[L^{k}, L\right], \quad k \geq 1 .
$$

Definition 2.2. A Leibniz algebra $\mathrm{L}$ is nilpotent if there exists an integer $s \in N$, such that

$$
L^{1} \supset L^{2} \supset \cdots \supset L^{s}=\{0\} .
$$

Definition 2.3. A Leibniz algebra $L$ is filiform if $\operatorname{dim} L^{i}=n-i$, where $n=\operatorname{dim} L$ and $2 \leq i \leq n$.

Obviously, a filiform Leibniz algebra is nilpotent. Throughout the paper all algebras are assumed to be over the field of complex numbers $\mathbb{C}$.

\section{Simplifications in $C E\left(\mu_{n}\right)$}

In this section we consider a subclass of the class of Leibniz algebras called truncated filiform Leibniz algebras in [6], which provided the motivation to study this case. The multiplication tables of the truncated filiform Leibniz algebras in the class $C E\left(\mu_{n}\right)$ can be represented as follows:

$$
\begin{aligned}
& {\left[e_{i}, e_{0}\right]=e_{i+1}, \quad 1 \leq i \leq n-1,} \\
& {\left[e_{0}, e_{i}\right]=-e_{i+1}, \quad 2 \leq i \leq n-1,} \\
& {\left[e_{0}, e_{0}\right]=b_{0,0} e_{n} \text {, }} \\
& {\left[e_{0}, e_{1}\right]=-e_{2}+b_{0,1} e_{n},} \\
& {\left[e_{1}, e_{1}\right]=b_{1,1} e_{n} \text {, }} \\
& {\left[e_{i}, e_{j}\right]=(-1)^{i-1} b_{1, i+j-1} e_{n}, \quad 1 \leq i<j \leq n-1, i+j \text { odd, }} \\
& {\left[e_{i}, e_{j}\right]=-\left[e_{j}, e_{i}\right], \quad 1 \leq i<j \leq n-1,} \\
& {\left[e_{i}, e_{n-i}\right]=-\left[e_{n-i}, e_{i}\right]=(-1)^{i} b e_{n}, \quad 1 \leq i \leq n-1 \text {, where } b=0 \text { for even } n \text {. }}
\end{aligned}
$$

The basis $\left\{e_{0}, e_{1}, \ldots, e_{n}\right\}$ is said to be adapted. Here are a few remarks about the multiplication table above.

(1) The undefined brackets are assumed to be zero.

(2) As a result of the Leibniz identity one has

$$
b_{i+1, j}=-b_{i, j+1}, \quad 1 \leq i, j \leq n-1, i+j \neq n,
$$

and

$$
b_{1,2 i+1}=0, \quad 0<i<\left[\frac{n-2}{2}\right] .
$$

As an immediate consequence of these relations we get

$$
b_{i+2, i}=b_{i, i+2}=0
$$


and

$$
\begin{aligned}
b_{i, j} & =-b_{i-1, j+1}=b_{i-2, j+2}=\cdots=(-1)^{i-1} b_{i-(i-1), j+(i-1)} \\
& =(-1)^{i-1} b_{1, j+i-1}
\end{aligned}
$$

(3) The centre of $L \in C E\left(\mu_{n}\right)$ is $\left\langle e_{n}\right\rangle$ and the quotient $L /\left\langle e_{n}\right\rangle$ is isomorphic to $\mu_{n}$. Hence, one can choose a Leibniz cocycle $\theta$ on $\mu_{n}$ such that $L$ is isomorphic to $L_{\theta}$. (The procedure is the same as in the Lie algebras case (see [2]).)

Elements of $C E\left(\mu_{n}\right)$ represented by the table above are denoted by $L(\alpha)$, where

$$
\alpha= \begin{cases}\left(b_{0,0}, b_{0,1}, b_{1,1}, b_{1,2}, b_{1,4}, \ldots, b_{1, n-2}\right) & \text { for even } n \\ \left(b_{0,0}, b_{0,1}, b_{1,1}, b_{1,2}, b_{1,4}, \ldots, b_{1, n-3}, b\right) & \text { for odd } n\end{cases}
$$

Since a truncated filiform Leibniz algebra admits an adapted basis it is sufficient to consider only base changes sending adapted bases to adapted. This set is a subgroup $G_{\text {ad }}$ of all base changes in $C E\left(\mu_{n}\right)$. A filiform Leibniz algebra is two-generated and so a base change on it can be given as:

$$
f\left(e_{0}\right)=\sum_{i=0}^{n} A_{i} e_{i}, \quad f\left(e_{1}\right)=\sum_{i=0}^{n} B_{i} e_{i}
$$

Then one has the following proposition.

Proposition 3.1. Let $f \in G_{\mathrm{ad}}$ and $L \in C E\left(\mu_{n}\right)$ then $f$ has the following form:

$$
\begin{aligned}
& e_{0}^{\prime}=f\left(e_{0}\right)=\sum_{i=0}^{n} A_{i} e_{i}, \\
& e_{i}^{\prime}=f\left(e_{i}\right)=\sum_{k=i}^{n-1} A_{0}^{i-1} B_{k-i+1} e_{i}+(*) e_{n}, \quad 1 \leq i \leq n-1, \\
& e_{n}^{\prime}=f\left(e_{n}\right)=A_{0}^{n-2} B_{1}\left(A_{0}+A_{1} b\right) e_{n},
\end{aligned}
$$

where $A_{0} B_{1}\left(A_{0}+A_{1} b\right) \neq 0$.

PROOF. It is easy to see that

$$
\begin{aligned}
& e_{i}^{\prime}=f\left(e_{i}\right)=\left[f\left(e_{i-1}\right), f\left(e_{0}\right)\right] \\
&=\sum_{j=i}^{n-1} A_{0}^{i-2}\left(A_{0} B_{j-i+1}-A_{j-i+1} B_{0}\right) e_{j}+(*) e_{n}, \quad 2 \leq i \leq n-1, \\
& e_{n}^{\prime}=f\left(e_{n}\right)=\left[f\left(e_{n-1}\right), f\left(e_{0}\right)\right]=A_{0}^{n-3}\left(A_{0} B_{1}-A_{1} B_{0}\right)\left(A_{0}+A_{1} b\right) e_{n} .
\end{aligned}
$$


Bearing in mind that $f$ is adapted, we get

$$
\begin{aligned}
{\left[f\left(e_{1}\right), f\left(e_{2}\right)\right] } & =b_{1,2}^{\prime} e_{n}^{\prime}=b_{1,2}^{\prime} A_{0}^{n-3}\left(A_{0} B_{1}-A_{1} B_{0}\right)\left(A_{0}+A_{1} b\right) e_{n} \\
& =B_{0} \sum_{i=3}^{n-1}\left(A_{0} B_{i-2}-A_{i-2} B_{0}\right) e_{i}+(*) e_{n} .
\end{aligned}
$$

Therefore,

$$
B_{0}\left(A_{0} B_{i-2}-A_{i-2} B_{0}\right)=0, \quad 3 \leq i \leq n-1 .
$$

Since $f$ is not singular, $A_{0} B_{1}-A_{1} B_{0} \neq 0$, and this implies that $B_{0}=0$.

Definition 3.2. The following types of adapted base change of $L \in C E\left(\mu_{n}\right)$ are said to be elementary:

$$
\begin{aligned}
\tau(a, b, c)= \begin{cases}\tau\left(e_{0}\right)=a e_{0}+b e_{1}, \\
\tau\left(e_{1}\right)=c e_{1}, & a c \neq 0, \\
\tau\left(e_{i+1}\right)=\left[\tau\left(e_{i}\right), \tau\left(e_{0}\right)\right], & 1 \leq i \leq n-1,\end{cases} \\
\sigma(a, k)= \begin{cases}\sigma\left(e_{0}\right)=e_{0}+a e_{k}, & 2 \leq k \leq n, \\
\sigma\left(e_{1}\right)=e_{1}, & \\
\sigma\left(e_{i+1}\right)=\left[\sigma\left(e_{i}\right), \sigma\left(e_{0}\right)\right], & 1 \leq i \leq n-1,\end{cases} \\
\phi(a, k)= \begin{cases}\phi\left(e_{0}\right)=e_{0}, \\
\phi\left(e_{1}\right)=e_{1}+c e_{k}, \\
\phi\left(e_{i+1}\right)=\left[\phi\left(e_{i}\right), \phi\left(e_{0}\right)\right], & 1 \leq i \leq n-1,\end{cases}
\end{aligned}
$$

where $a, b, c \in \mathbb{C}$.

PROPOSITION 3.3. Let $f$ be an adapted transformation of $L \in C E\left(\mu_{n}\right)$. Then it can be represented as the composition

$$
\begin{aligned}
f=\phi & \left(B_{n}, n\right) \circ \phi\left(B_{n-1}, n-1\right) \circ \cdots \circ \phi\left(B_{2}, 2\right) \circ \sigma\left(A_{n}, n\right) \circ \sigma\left(A_{n-1}, n-1\right) \circ \cdots \\
& \circ \sigma\left(A_{2}, 2\right) \circ \tau\left(A_{0}, A_{1}, B_{1}\right) .
\end{aligned}
$$

PROOF. The proof is straightforward.

\section{PROPOSITION 3.4. The adapted transformation}

$$
\begin{aligned}
g=\phi & \left(B_{n}, n\right) \circ \phi\left(B_{n-1}, n-1\right) \circ \phi\left(B_{n-2}, n-2\right) \\
& \circ \sigma\left(A_{n}, n\right) \circ \sigma\left(A_{n-1}, n-1\right) \circ \cdots \circ \sigma\left(A_{2}, 2\right),
\end{aligned}
$$

for even $n$, and

$$
g=\phi\left(B_{n}, n\right) \circ \phi\left(B_{n-1}, n-1\right) \circ \sigma\left(A_{n}, n\right) \circ \sigma\left(A_{n-1}, n-1\right) \circ \cdots \circ \sigma\left(A_{2}, 2\right),
$$

for odd $n$, does not change the structure constants of $L \in C E\left(\mu_{n}\right)$. 
PROOF. Consider the transformation

$$
\sigma\left(A_{k}, k\right)= \begin{cases}\sigma\left(e_{0}\right)=e_{0}+A_{k} e_{k}, & 2 \leq k \leq n \\ \sigma\left(e_{1}\right)=e_{1}, & \\ \sigma\left(e_{i+1}\right)=\left[\sigma\left(e_{i}\right), \sigma\left(e_{0}\right)\right], & 1 \leq i \leq n-1 .\end{cases}
$$

First notice that

$$
\sigma\left(e_{i}\right)=e_{i}+(-1)^{i} \delta(i, k) A_{k} b_{1, i+k-2} e_{n}, \quad 2 \leq i \leq n-1,
$$

where

$$
\delta(s, t)= \begin{cases}1 & \text { if } s+t \text { is even } \\ -1 & \text { if } s=n-1 \\ 0 & \text { otherwise }\end{cases}
$$

Indeed,

$$
\begin{aligned}
{\left[\sigma\left(e_{i}\right), \sigma\left(e_{j}\right)\right] } & =\left[e_{i}+(-1)^{i} \delta(i, k) A_{k} b_{1, i+k-2} e_{n}, e_{j}+(-1)^{j} \delta(j, k) A_{k} b_{1, j+k-2} e_{n}\right] \\
& =\left[e_{i}, e_{j}\right] .
\end{aligned}
$$

The calculations for $\phi\left(B_{n}, n\right)$ and $\phi\left(B_{n-1}, n-1\right)$ are handled similarly. It is worth mentioning that for even $n$ the transformation $\phi\left(B_{n-2}, n-2\right)$ does not change the structure constants, because in this case $b=0$.

The next lemma keeps track of the behaviour of the structure constants of algebras from $C E\left(\mu_{n}\right)$ under the adapted basis change.

LEMMA 3.5. Let $L(\alpha) \in C E\left(\mu_{n}\right)$ and $L\left(\alpha^{\prime}\right)$ be the image of $L(\alpha)$ under the action of $G_{\text {ad. Then one has }}$

$$
\begin{aligned}
b_{0,0}^{\prime}= & \frac{A_{0}^{2} b_{0,0}+A_{0} A_{1} b_{0,1}+A_{1}^{2} b_{1,1}}{A_{0}^{n-2} B_{1}\left(A_{0}+A_{1} b\right)}, \quad b_{0,1}^{\prime}=\frac{A_{0} b_{0,1}+2 A_{1} b_{1,1}}{A_{0}^{n-2}\left(A_{0}+A_{1} b\right)}, \\
b_{1,1}^{\prime}= & \frac{B_{1} b_{1,1}}{A_{0}^{n-2}\left(A_{0}+A_{1} b\right)}, \\
b_{1,2 j}^{\prime}= & \frac{1}{A_{0}^{n-2 j-1} B_{1}\left(A_{0}+A_{1} b\right)} \\
b^{\prime}= & \frac{B_{1} b}{A_{0}+A_{1} b} .
\end{aligned}
$$


PROOF. Consider the product $\left[f\left(e_{0}\right), f\left(e_{0}\right)\right]=b_{0,0}^{\prime} f\left(e_{n}\right)$. Equating coefficients of $e_{n}$ in it, we get

$$
A_{0}^{2} b_{0,0}+A_{0} A_{1} b_{0,1}+A_{1}^{2} b_{1,1}=b_{0,0}^{\prime} A_{0}^{n-2} B_{1}\left(A_{0}+A_{1} b\right) .
$$

Then we find

$$
b_{0,0}^{\prime}=\frac{A_{0}^{2} b_{0,0}+A_{0} A_{1} b_{0,1}+A_{1}^{2} b_{1,1}}{A_{0}^{n-2} B_{1}\left(A_{0}+A_{1} b\right)} .
$$

The product $\left[f\left(e_{1}\right), f\left(e_{1}\right)\right]=b_{1,1}^{\prime} f\left(e_{n}\right)$ yields $b_{1,1}^{\prime}=B_{1} b_{1,1} / A_{0}^{n-2}\left(A_{0}+A_{1} b\right)$. Analysing the equality

$$
b_{0,1}^{\prime} f\left(e_{n}\right)=\left[f\left(e_{1}\right), f\left(e_{0}\right)\right]+\left[f\left(e_{0}\right), f\left(e_{1}\right)\right],
$$

we obtain $b_{0,1}^{\prime} A_{0}^{n-2} B_{1}\left(A_{0}+A_{1} b\right)=A_{0} B_{1} b_{0,1}+2 A_{1} B_{1} b_{1,1}$, and this implies that

$$
b_{0,1}^{\prime}=\frac{A_{0} b_{0,1}+2 A_{1} b_{1,1}}{A_{0}^{n-2}\left(A_{0}+A_{1} b\right)} .
$$

From Propositions 3.3 and 3.4,

$$
\begin{aligned}
e_{0}^{\prime} & =A_{0} e_{0}+A_{1} e_{1}, \\
e_{1}^{\prime} & =B_{1} e_{1}+B_{2} e_{2}+\cdots+B_{n-2} e_{n-2}, \\
e_{i}^{\prime} & =\sum_{k=i}^{n-1} A_{0}^{i-1} B_{k-i+1} e_{i}+(*) e_{n}, \quad 2 \leq i \leq n-1, \\
e_{n}^{\prime} & =A_{0}^{n-2} B_{1}\left(A_{0}+A_{1} b\right) e_{n} .
\end{aligned}
$$

Then

$$
\begin{aligned}
{\left[e_{i}^{\prime}, e_{j}^{\prime}\right] } & =\left[\sum_{k=i}^{n-1} A_{0}^{i-1} B_{k-i+1} e_{k}+(*) e_{n}, \sum_{l=j}^{n-1} A_{0}^{j-1} B_{l-j+1} e_{l}+(*) e_{n}\right] \\
& =\left[\sum_{k=i}^{n-1} A_{0}^{i-1} B_{k-i+1} e_{k}, \sum_{l=j}^{n-1} A_{0}^{j-1} B_{l-j+1} e_{l}\right] \\
& =\sum_{k=i}^{n-1} \sum_{l=j}^{n-1} A_{0}^{i+j-2} B_{k-i+1} B_{l-j+1}\left[e_{k}, e_{l}\right] \\
& =\sum_{k=i}^{n-1} \sum_{l=j}^{n-k} A_{0}^{i+j-2} B_{k-i+1} B_{l-j+1} b_{k, l} e_{n} .
\end{aligned}
$$

Hence the equality $b_{i, j}^{\prime} e_{n}^{\prime}=\left[e_{i}^{\prime}, e_{j}^{\prime}\right]$ gives the relation

$$
b_{i, j}^{\prime} A_{0}^{n-2} B_{1}\left(A_{0}+A_{1} b\right)=A_{0}^{i+j-2} \sum_{k=i}^{n-1} \sum_{l=j}^{n-k} B_{k-i+1} B_{l-j+1} b_{k, l},
$$


and implies that

$$
b_{i, j}^{\prime}=\frac{1}{A_{0}^{n-i-j} B_{1}\left(A_{0}+A_{1} b\right)} \sum_{k=i}^{n-1} \sum_{l=j}^{n-k} B_{k-i+1} B_{l-j+1} b_{k, l} .
$$

Now use (3.2) to obtain

$$
\begin{aligned}
b_{1,2 j}^{\prime}= & \frac{1}{A_{0}^{n-2 j-1} B_{1}\left(A_{0}+A_{1} b\right)} \\
& \quad \times\left(\sum_{k=1}^{n-1} \sum_{\substack{l=2 j \\
l+k \neq n}}^{n-k-1}(-1)^{k-1} B_{k} B_{l-2 j+1} b_{1, k+l-1}+\sum_{k=1}^{n-2}(-1)^{k} B_{k} B_{n-k-2 j+1} b\right) .
\end{aligned}
$$

Finally, the last equality comes from $\left[e_{n-1}^{\prime}, e_{1}^{\prime}\right]=b^{\prime} e_{n}^{\prime}$.

The next sections deal with the applications of the results of this section to the classification problem of $C E\left(\mu_{n}\right)$ for $n=4, \ldots, 8$. We remind the reader that the classification of all complex nilpotent Leibniz algebras in dimensions at most four has been given before in [1].

Here, to classify algebras from the class $C E\left(\mu_{n}\right)$ in each fixed dimension we represent $C E\left(\mu_{n}\right)$ as a disjoint union of its subsets. Some of these subsets are single orbits and others contain infinitely many orbits. In the last case we give invariant functions to distinguish the orbits.

To simplify calculations we introduce the following notation:

$$
\Delta=b_{0,1}^{2}-4 b_{0,0} b_{1,1} \quad \text { and } \quad \Delta^{\prime}=b_{0,1}^{\prime 2}-4 b_{0,0}^{\prime} b_{1,1}^{\prime} .
$$

3.1. Isomorphism classes in $\boldsymbol{C E}\left(\boldsymbol{\mu}_{4}\right)$. In this section we describe the isomorphism classes of algebras from $C E\left(\mu_{4}\right)$. According to the notation introduced above, elements of $C E\left(\mu_{4}\right)$ will be denoted by $L(\alpha)$, where $\alpha=\left(b_{0,0}, b_{0,1}, b_{1,1}, b_{1,2}\right)$. Note that in this case $b=0$ because $n$ is even (see the multiplication table of $C E\left(\mu_{n}\right)$ ).

THEOREM 3.6 (Isomorphism criterion for $C E\left(\mu_{4}\right)$ ). Two filiform Leibniz algebras $L(\alpha)$ and $L\left(\alpha^{\prime}\right)$ from $C E\left(\mu_{4}\right)$ are isomorphic if and only if there exist $A_{0}, A_{1}, B_{1} \in \mathbb{C}$ such that $A_{0} B_{1} \neq 0$ and the following equalities hold:

$$
\begin{aligned}
b_{0,0}^{\prime} & =\frac{A_{0}^{2} b_{0,0}+A_{0} A_{1} b_{0,1}+A_{1}^{2} b_{1,1}}{A_{0}^{3} B_{1}} \\
b_{1,1}^{\prime} & =\frac{B_{1} b_{1,1}}{A_{0}^{3}} \\
b_{0,1}^{\prime} & =\frac{A_{0} b_{0,1}+2 A_{1} b_{1,1}}{A_{0}^{3}} \\
b_{1,2}^{\prime} & =\frac{B_{1} b_{1,2}}{A_{0}^{2}}
\end{aligned}
$$


PROOF. If $L(\alpha)$ and $L\left(\alpha^{\prime}\right)$ are isomorphic, then the equalities are a consequence of Lemma 3.5.

Conversely, suppose that the equalities (3.5)-(3.8) hold. Then the following base change is adapted and it transforms $L(\alpha)$ to $L\left(\alpha^{\prime}\right)$ :

$$
\begin{aligned}
& e_{0}^{\prime}=A_{0} e_{0}+A_{1} e_{1}, \\
& e_{1}^{\prime}=B_{1} e_{1}, \\
& e_{2}^{\prime}=A_{0} B_{1} e_{2}+A_{1} B_{1} b_{1,1} e_{4}, \\
& e_{3}^{\prime}=A_{0}^{2} B_{1} e_{3}-A_{1} A_{0} B_{1} b_{1,2} e_{4}, \\
& e_{4}^{\prime}=A_{0}^{3} B_{1} e_{4} .
\end{aligned}
$$

Indeed,

$$
\begin{aligned}
{\left[e_{0}^{\prime}, e_{0}^{\prime}\right] } & =A_{0}^{2} b_{0,0} e_{4}+A_{0} A_{1}\left(-e_{2}+b_{0,1} e_{4}\right)+A_{1} A_{0} e_{2}+A_{1}^{2} b_{1,1} e_{4} \\
& =\frac{A_{0}^{2} b_{0,0}+A_{0} A_{1} b_{0,1}+A_{1}^{2} b_{1,1}}{A_{0}^{3} B_{1}} A_{0}^{3} B_{1} e_{4}=b_{0,0}^{\prime} e_{4}^{\prime} .
\end{aligned}
$$

Similarly, one easily can see that

$$
\begin{aligned}
{\left[e_{0}^{\prime}, e_{1}^{\prime}\right] } & =-A_{0} B_{1} e_{2}+A_{0} B_{1} b_{0,1} e_{4}+A_{1} B_{1} b_{1,1} e_{4} \\
& =-A_{0} B_{1} e_{2}-A_{1} B_{1} b_{1,1} e_{4}+A_{0} B_{1} b_{0,1} e_{4}+2 A_{1} B_{1} b_{1,1} e_{4} \\
& =-e_{2}^{\prime}+B_{1}\left(A_{0} b_{0,1}+2 A_{1} b_{1,1}\right) e_{4} \\
& =-e_{2}^{\prime}+b_{0,1}^{\prime} A_{0}^{3} B_{1} e_{4}=-e_{2}^{\prime}+b_{0,1}^{\prime} e_{4}^{\prime} \\
{\left[e_{1}^{\prime}, e_{1}^{\prime}\right] } & =B_{1}^{2} b_{1,1} e_{4}=A_{0}^{3} B_{1} b_{1,1}^{\prime} e_{4}=b_{1,1}^{\prime} e_{4}, \\
{\left[e_{1}^{\prime}, e_{2}^{\prime}\right] } & =B_{1}^{2} A_{0} b_{1,2} e_{4}=A_{0}^{3} B_{1} b_{1,2}^{\prime} e_{4}=b_{1,2}^{\prime} e_{4} .
\end{aligned}
$$

The other products of the basis vectors $e_{0}^{\prime}, e_{1}^{\prime}, \ldots, e_{n}^{\prime}$ are zero.

In order to describe the isomorphism classes of algebras from $C E\left(\mu_{4}\right)$ we represent it as a disjoint union of the following subsets:

$$
\begin{aligned}
U_{4}^{1} & =\left\{L(\alpha) \in C E\left(\mu_{4}\right) \mid b_{1,1} \neq 0, b_{1,2} \neq 0\right\} ; \\
U_{4}^{2} & =\left\{L(\alpha) \in C E\left(\mu_{4}\right) \mid b_{1,1} \neq 0, b_{1,2}=0, \Delta \neq 0\right\} ; \\
U_{4}^{3} & =\left\{L(\alpha) \in C E\left(\mu_{4}\right) \mid b_{1,1} \neq 0, b_{1,2}=\Delta=0\right\} ; \\
U_{4}^{4} & =\left\{L(\alpha) \in C E\left(\mu_{4}\right) \mid b_{1,1}=0, b_{0,1} \neq 0, b_{1,2} \neq 0\right\} ; \\
U_{4}^{5} & =\left\{L(\alpha) \in C E\left(\mu_{4}\right) \mid b_{1,1}=0, b_{0,1} \neq 0, b_{1,2}=0\right\} ; \\
U_{4}^{6} & =\left\{L(\alpha) \in C E\left(\mu_{4}\right) \mid b_{1,1}=b_{0,1}=0, b_{0,0} \neq 0, b_{1,2} \neq 0\right\} ; \\
U_{4}^{7} & =\left\{L(\alpha) \in C E\left(\mu_{4}\right) \mid b_{1,1}=b_{0,1}=0, b_{0,0} \neq 0, b_{1,2}=0\right\} ; \\
U_{4}^{8} & =\left\{L(\alpha) \in C E\left(\mu_{4}\right) \mid b_{1,1}=b_{0,1}=b_{0,0}=0, b_{1,2} \neq 0\right\} ; \\
U_{4}^{9} & =\left\{L(\alpha) \in C E\left(\mu_{4}\right) \mid b_{1,1}=b_{0,1}=b_{0,0}=b_{1,2}=0\right\} .
\end{aligned}
$$


Here, the subset $U_{4}^{1}$ turns out to be a union of infinitely many orbits. The following proposition is a description of $U_{4}^{1}$.

PROPOSITION 3.7.

(1) Two algebras $L(\alpha)$ and $L\left(\alpha^{\prime}\right)$ from $U_{4}^{1}$ are isomorphic if and only if

$$
\left(\frac{b_{1,2}^{\prime}}{b_{1,1}^{\prime}}\right)^{4} \Delta^{\prime}=\left(\frac{b_{1,2}}{b_{1,1}}\right)^{4} \Delta .
$$

(2) For any $\lambda$ from $\mathbb{C}$ there exists $L(\alpha) \in U_{4}^{1}$ such that $\left(b_{1,2} / b_{1,1}\right)^{4} \Delta=\lambda$.

Proof. (1) Let $L(\alpha)$ and $L\left(\alpha^{\prime}\right)$ be isomorphic. Then from Theorem 3.6 there are complex numbers $A_{0}, A_{1}$ and $B_{1}$ such that $A_{0} B_{1} \neq 0$ and the action of the adapted group $G_{\mathrm{ad}}$ is expressed by the following system of equalities:

$$
\begin{aligned}
& b_{0,0}^{\prime}=\frac{A_{0}^{2} b_{0,0}+A_{0} A_{1} b_{0,1}+A_{1}^{2} b_{1,1}}{A_{0}^{3} B_{1}} \\
& b_{1,1}^{\prime}=\frac{B_{1} b_{1,1}}{A_{0}^{3}}, \\
& b_{0,1}^{\prime}=\frac{A_{0} b_{0,1}+2 A_{1} b_{1,1}}{A_{0}^{3}} \\
& b_{1,2}^{\prime}=\frac{B_{1}}{A_{0}^{2}} b_{1,2} .
\end{aligned}
$$

Then one can easily see that $\left(b_{1,2}^{\prime} / b_{1,1}^{\prime}\right)^{4} \Delta^{\prime}=\left(b_{1,2} / b_{1,1}\right)^{4} \Delta$.

Conversely, suppose that that the equality $\left(b_{1,2}^{\prime} / b_{1,1}^{\prime}\right)^{4} \Delta^{\prime}=\left(b_{1,2} / b_{1,1}\right)^{4} \Delta$ holds.

Let $\left\{e_{0}, e_{1}, e_{2}, e_{3}, e_{4}\right\}$ and $\left\{e_{0}^{\prime}, e_{1}^{\prime}, e_{2}^{\prime}, e_{3}^{\prime}, e_{4}^{\prime}\right\}$ be adapted bases of $L(\alpha)$ and $L\left(\alpha^{\prime}\right)$, respectively. Then the adapted base change

$$
\begin{aligned}
& f_{0}=A_{0} e_{0}+A_{1} e_{1}, \\
& f_{1}=B_{1} e_{1}, \\
& f_{2}=A_{0} B_{1} e_{2}+A_{1} B_{1} b_{1,1} e_{4}, \\
& f_{3}=A_{0}^{2} B_{1} e_{3}-A_{1} A_{0} B_{1} b_{1,2} e_{4}, \\
& f_{4}=A_{0}^{3} B_{1} e_{4},
\end{aligned}
$$

where $A_{0}=b_{1,1} / b_{1,2}, A_{1}=-b_{0,1} / 2 b_{1,2}$ and $B_{1}=b_{1,1}^{2} / b_{1,2}^{3}$, transforms $L(\alpha)$ to $L\left(\left(b / 1,2 b_{1,1}\right)^{4} \Delta, 0,1,1\right)$.

An analogous base change

$$
\begin{aligned}
& f_{0}^{\prime}=A_{0}^{\prime} e_{0}^{\prime}+A_{1}^{\prime} e_{1}^{\prime}, \\
& f_{1}^{\prime}=B_{1}^{\prime} e_{1}^{\prime}, \\
& f_{2}^{\prime}=A_{0}^{\prime} B_{1}^{\prime} e_{2}^{\prime}+A_{1}^{\prime} B_{1}^{\prime} b_{1,1}^{\prime} e_{4}^{\prime},
\end{aligned}
$$




$$
\begin{aligned}
& f_{3}^{\prime}=A_{0}^{\prime 2} B_{1}^{\prime} e_{3}^{\prime}-A_{1}^{\prime} A_{0}^{\prime} B_{1}^{\prime} b_{1,2}^{\prime} e_{4}^{\prime}, \\
& f_{4}^{\prime}=A_{0}^{\prime 3} B_{1}^{\prime} e_{4}^{\prime},
\end{aligned}
$$

where $A_{0}^{\prime}=b_{1,1}^{\prime} / b_{1,2}^{\prime}, A_{1}^{\prime}=-b_{0,1}^{\prime} / 2 b_{1,2}^{\prime}$ and $B_{1}^{\prime}=b /_{1,1}^{\prime 2} b_{1,2}^{\prime 3}$, transforms $L\left(\alpha^{\prime}\right)$ to $L\left(\left(b_{1,2}^{\prime} / b_{1,1}^{\prime}\right)^{4} \Delta^{\prime}, 0,1,1\right)$. However, by hypothesis, $\left(b_{1,2} / b_{1,1}\right)^{4} \Delta=\left(b_{1,2}^{\prime} / b_{1,1}^{\prime}\right)^{4} \Delta^{\prime}$. Hence $L(\alpha)$ and $L\left(\alpha^{\prime}\right)$ are isomorphic.

(2) This is obvious.

As a consequence of this proposition the orbits in $U_{4}^{1}$ can be parameterized as $L(\lambda, 0,1,1), \lambda \in \mathbb{C}$.

The next proposition is a description of single orbits.

PROPOSITION 3.8. The subsets $U_{4}^{2}, U_{4}^{3}, U_{4}^{4}, U_{4}^{5}, U_{4}^{6}, U_{4}^{7}, U_{4}^{8}$ and $U_{4}^{9}$ are single orbits with representatives

$$
\begin{gathered}
L(1,0,1,0), \quad L(0,0,1,0), \quad L(0,1,0,1), \quad L(0,1,0,0), \\
L(1,0,0,1), \quad L(1,0,0,0), \quad L(0,0,0,1) \text { and } \quad L(0,0,0,0),
\end{gathered}
$$

respectively.

PROOF. For each of the subsets above we give the corresponding base change leading to the indicated representative.

For $U_{4}^{2}$ :

$$
\begin{gathered}
e_{0}^{\prime}=A_{0} e_{0}+A_{1} e_{1}, \quad e_{1}^{\prime}=B_{1} e_{1}, \quad e_{2}^{\prime}=A_{0} B_{1} e_{2}+A_{1} B_{1} b_{1,1} e_{4}, \\
e_{3}^{\prime}=A_{0}^{2} B_{1} e_{3}, \quad e_{4}^{\prime}=A_{0}^{3} B_{1} e_{4},
\end{gathered}
$$

where

$$
A_{0}^{4}=\frac{\Delta}{4}, \quad A_{1}^{4}=\frac{b_{0,1}^{4} \Delta}{64 b_{1,1}^{4}} \quad \text { and } \quad B_{1}^{4}=\frac{\Delta^{3}}{64 b_{1,1}^{4}}
$$

For $U_{4}^{3}$ :

$$
\begin{gathered}
e_{0}^{\prime}=A_{0} e_{0}+A_{1} e_{1}, \quad e_{1}^{\prime}=B_{1} e_{1}, \quad e_{2}^{\prime}=A_{0} B_{1} e_{2}+A_{1} B_{1} b_{1,1} e_{4}, \\
e_{3}^{\prime}=A_{0}^{2} B_{1} e_{3}, \quad e_{4}^{\prime}=A_{0}^{3} B_{1} e_{4},
\end{gathered}
$$

where

$$
A_{0} \in \mathbb{C}^{*}, \quad A_{1}=\frac{-A_{0} b_{0,1}}{2 b_{1,1}} \quad \text { and } \quad B_{1}=\frac{A_{0}^{3}}{b_{1,1}}
$$

For $U_{4}^{4}$ :

$$
\begin{gathered}
e_{0}^{\prime}=A_{0} e_{0}+A_{1} e_{1}, \quad e_{1}^{\prime}=B_{1} e_{1}, \quad e_{2}^{\prime}=A_{0} B_{1} e_{2}, \\
e_{3}^{\prime}=A_{0}^{2} B_{1} e_{3}-A_{0} A_{1} B_{1} b_{1,2} e_{4},
\end{gathered}
$$


where

$$
A_{0}^{2}=b_{0,1}, \quad A_{1}^{2}=\frac{b_{0,0}^{2}}{b_{0,1}} \quad \text { and } \quad B_{1}=\frac{b_{0,1}}{b_{1,2}} .
$$

For $U_{4}^{5}$ :

$e_{0}^{\prime}=A_{0} e_{0}+A_{1} e_{1}, \quad e_{1}^{\prime}=B_{1} e_{1}, \quad e_{2}^{\prime}=A_{0} B_{1} e_{2}, \quad e_{3}^{\prime}=A_{0}^{2} B_{1} e_{3}, \quad e_{4}^{\prime}=A_{0}^{3} B_{1} e_{4}$,

where

$$
A_{0}^{2}=b_{0,1}, \quad A_{1}^{2}=\frac{b_{0,0}^{2}}{b_{0,1}} \quad \text { and } \quad B_{1} \in \mathbb{C}^{*} .
$$

For $U_{4}^{6}$ :

$$
\begin{gathered}
e_{0}^{\prime}=A_{0} e_{0}+A_{1} e_{1}, \quad e_{1}^{\prime}=B_{1} e_{1}, \quad e_{2}^{\prime}=A_{0} B_{1} e_{2} \\
e_{3}^{\prime}=A_{0}^{2} B_{1} e_{3}-A_{0} A_{1} B_{1} b_{1,2} e_{4}, \quad e_{4}^{\prime}=A_{0}^{3} B_{1} e_{4}
\end{gathered}
$$

where

$$
A_{0}^{3}=b_{0,0} b_{1,2}, \quad A_{1} \in \mathbb{C} \quad \text { and } \quad B_{1}^{3}=\frac{b_{0,0}^{3}}{b_{0,0} b_{1,2}}
$$

For $U_{4}^{7}$ :

$e_{0}^{\prime}=A_{0} e_{0}+A_{1} e_{1}, \quad e_{1}^{\prime}=B_{1} e_{1}, \quad e_{2}^{\prime}=A_{0} B_{1} e_{2}, \quad e_{3}^{\prime}=A_{0}^{2} B_{1} e_{3}, \quad e_{4}^{\prime}=A_{0}^{3} B_{1} e_{4}$,

where

$$
A_{0} \in \mathbb{C}^{*}, \quad A_{1} \in \mathbb{C} \quad \text { and } \quad B_{1}=\frac{b_{0,0}}{A_{0}} .
$$

For $U_{4}^{8}$ :

$$
\begin{gathered}
e_{0}^{\prime}=A_{0} e_{0}+A_{1} e_{1}, \quad e_{1}^{\prime}=B_{1} e_{1}, \quad e_{2}^{\prime}=A_{0} B_{1} e_{2} \\
e_{3}^{\prime}=A_{0}^{2} B_{1} e_{3}-A_{0} A_{1} B_{1} b_{1,2} e_{4}, \quad e_{4}^{\prime}=A_{0}^{3} B_{1} e_{4}
\end{gathered}
$$

where

$$
A_{0} \in \mathbb{C}^{*}, \quad A_{1} \in \mathbb{C} \quad \text { and } \quad B_{1}=\frac{A_{0}^{2}}{b_{1,2}} .
$$

For $U_{4}^{9}$ :

$$
e_{0}^{\prime}=A_{0} e_{0}+A_{1} e_{1}, \quad e_{1}^{\prime}=B_{1} e_{1}, \quad e_{2}^{\prime}=A_{0} B_{1} e_{2}, \quad e_{3}^{\prime}=A_{0}^{2} B_{1} e_{3}, \quad e_{4}^{\prime}=A_{0}^{3} B_{1} e_{4},
$$

where

$$
A_{0}, B_{1} \in \mathbb{C}^{*}, \quad A_{1} \in \mathbb{C}
$$

This concludes the proof. 
3.2. Isomorphism classes in $\boldsymbol{C E}\left(\boldsymbol{\mu}_{5}\right)$. Notice that in this case $b=b_{2,3}=b_{1,4}$. Hence, an element of $C E\left(\mu_{5}\right)$ is $L(\alpha)$, with $\alpha=\left(b_{0,0}, b_{0,1}, b_{1,1}, b_{1,2}, b\right)$.

THEOREM 3.9 (Isomorphism criterion for $C E\left(\mu_{5}\right)$ ). Two filiform Leibniz algebras $L(\alpha)$ and $L\left(\alpha^{\prime}\right)$ from $C E\left(\mu_{5}\right)$ are isomorphic if and only if there exist $A_{0}, A_{1}, B_{1}, B_{2}, B_{3} \in \mathbb{C}$ such that $A_{0} B_{1}\left(A_{0}+A_{1} b\right) \neq 0$, and the following equalities hold:

$$
\begin{aligned}
b_{0,0}^{\prime} & =\frac{A_{0}^{2} b_{0,0}+A_{0} A_{1} b_{0,1}+A_{1}^{2} b_{1,1}}{A_{0}^{3} B_{1}\left(A_{0}+A_{1} b\right)}, \\
b_{0,1}^{\prime} & =\frac{A_{0} b_{0,1}+2 A_{1} b_{1,1}}{A_{0}^{3}\left(A_{0}+A_{1} b\right)}, \\
b_{1,1}^{\prime} & =\frac{B_{1} b_{1,1}}{A_{0}^{3}\left(A_{0}+A_{1} b\right)}, \\
b_{1,2}^{\prime} & =\frac{B_{1}^{2} b_{1,2}+\left(B_{2}^{2}-2 B_{1} B_{3}\right) b}{A_{0}^{2} B_{1}\left(A_{0}+A_{1} b\right)}, \\
b^{\prime} & =\frac{B_{1} b}{A_{0}+A_{1} b} .
\end{aligned}
$$

To find the isomorphism classes in $C E\left(\mu_{5}\right)$ we represent it as a union of the following subsets:

$$
\begin{aligned}
U_{5}^{1} & =\left\{L(\alpha) \in C E\left(\mu_{5}\right) \mid b \neq 0, b_{1,1} \neq 0\right\} ; \\
U_{5}^{2} & =\left\{L(\alpha) \in C E\left(\mu_{5}\right) \mid b \neq 0, b_{1,1}=0, b_{0,1} \neq 0\right\} ; \\
U_{5}^{3} & =\left\{L(\alpha) \in C E\left(\mu_{5}\right) \mid b \neq 0, b_{1,1}=b_{0,1}=0, b_{0,0} \neq 0\right\} ; \\
U_{5}^{4} & =\left\{L(\alpha) \in C E\left(\mu_{5}\right) \mid b \neq 0, b_{1,1}=b_{0,1}=b_{0,0}=0\right\} ; \\
U_{5}^{5} & =\left\{L(\alpha) \in C E\left(\mu_{5}\right) \mid b=0, b_{1,1} \neq 0, b_{1,2} \neq 0\right\} ; \\
U_{5}^{6} & =\left\{L(\alpha) \in C E\left(\mu_{5}\right) \mid b=0, b_{1,1} \neq 0, b_{1,2}=0, \Delta \neq 0\right\} ; \\
U_{5}^{7} & =\left\{L(\alpha) \in C E\left(\mu_{5}\right) \mid b=0, b_{1,1} \neq 0, b_{1,2}=\Delta=0\right\} ; \\
U_{5}^{8} & =\left\{L(\alpha) \in C E\left(\mu_{5}\right) \mid b=b_{1,1}=0, b_{0,1} \neq 0, b_{1,2} \neq 0\right\} ; \\
U_{5}^{9} & =\left\{L(\alpha) \in C E\left(\mu_{5}\right) \mid b=b_{1,1}=0, b_{0,1} \neq 0, b_{1,2}=0\right\} ; \\
U_{5}^{10} & =\left\{L(\alpha) \in C E\left(\mu_{5}\right) \mid b=b_{1,1}=b_{0,1}=0, b_{0,0} \neq 0, b_{1,2} \neq 0\right\} ; \\
U_{5}^{11} & =\left\{L(\alpha) \in C E\left(\mu_{5}\right) \mid b=b_{1,1}=b_{0,1}=0, b_{0,0} \neq 0, b_{1,2}=0\right\} ; \\
U_{5}^{12} & =\left\{L(\alpha) \in C E\left(\mu_{5}\right) \mid b=b_{1,1}=b_{0,1}=b_{0,0}=0, b_{1,1} \neq 0\right\} ; \\
U_{5}^{13} & =\left\{L(\alpha) \in C E\left(\mu_{5}\right) \mid b=b_{1,1}=b_{0,1}=b_{0,0}=b_{1,1}=0\right\} .
\end{aligned}
$$

Here, the subsets $U_{5}^{1}$ and $U_{5}^{5}$ turn out to be a union of infinitely many orbits. The following propositions are descriptions of them. 
PROPOSITION 3.10.

(1) Two algebras $L(\alpha)$ and $L\left(\alpha^{\prime}\right)$ from $U_{5}^{1}$ are isomorphic if and only if

$$
\frac{\Delta^{\prime} b^{\prime 2}}{\left(b_{0,1}^{\prime} b^{\prime}-2 b_{1,1}^{\prime}\right)^{2}}=\frac{\Delta b^{2}}{\left(b_{0,1} b-2 b_{1,1}\right)^{2}}
$$

(2) For any $\lambda$ from $\mathbb{C}$, there exists $L(\alpha) \in U_{5}^{1}$ such that $\Delta b^{2} /\left(b_{0,1} b-2 b_{1,1}\right)^{2}=\lambda$.

The orbits in $U_{5}^{1}$ are parameterized as $L(\lambda, 0,1,0,1), \lambda \in \mathbb{C}$.

PROPOSITION 3.11.

(1) Two algebras $L(\alpha)$ and $L\left(\alpha^{\prime}\right)$ from $U_{5}^{5}$ are isomorphic if and only if

$$
\left(\frac{b_{1,2}^{\prime}}{b_{1,1}^{\prime}}\right)^{6} \Delta^{\prime}=\left(\frac{b_{1,2}}{b_{1,1}}\right)^{6} \Delta
$$

(2) For any $\lambda$ from $\mathbb{C}$ there exists $L(\alpha) \in U_{5}^{5}$ such that $\left(b_{1,2} / 4 b_{1,1}\right)^{6} \Delta=\lambda$.

The orbits in $U_{5}^{5}$ are parameterized as $L(\lambda, 0,1,1,0), \lambda \in \mathbb{C}$.

Proposition 3.12. The subsets $U_{5}^{2}, U_{5}^{3}, U_{5}^{4}, U_{5}^{6}, U_{5}^{7}, U_{5}^{8}, U_{5}^{9}, U_{5}^{10}, U_{5}^{11}, U_{5}^{12}$, and $U_{5}^{13}$ are single orbits with representatives

$$
\begin{gathered}
L(0,1,0,0,1), \quad L(1,0,0,0,1), \quad L(0,0,0,0,1), \quad L(1,0,1,0,0), \\
L(0,0,1,0,0), \quad L(0,1,0,1,0), \quad L(0,1,0,0,0), \quad L(1,0,0,1,0), \\
L(1,0,0,0,0), \quad L(0,0,0,1,0) \quad \text { and } L(0,0,0,0,0),
\end{gathered}
$$

respectively.

3.3. Isomorphism classes in $\boldsymbol{C E}\left(\boldsymbol{\mu}_{\mathbf{6}}\right)$. The section deals with the classification of $C E\left(\mu_{6}\right)$. It is easy to see here that $b_{2,3}=-b_{1,4}$.

THEOREM 3.13 (Isomorphism criterion for $C E\left(\mu_{6}\right)$ ). Two filiform Leibniz algebras $L(\alpha)$ and $L\left(\alpha^{\prime}\right), \alpha=\left(b_{0,0}, b_{0,1}, b_{1,1}, b_{1,2}, b_{1,4}\right)$ and $\alpha^{\prime}=\left(b_{0,0}^{\prime}, b_{0,1}^{\prime}, b_{1,1}^{\prime}, b_{1,2}^{\prime}, b_{1,4}^{\prime}\right)$, from $C E\left(\mu_{6}\right)$ are isomorphic if and only if there exist $A_{0}, A_{1}, B_{1}, B_{2}, B_{3} \in \mathbb{C}$ such that $A_{0} B_{1} \neq 0$ and the following equalities hold:

$$
\begin{aligned}
& b_{0,0}^{\prime}=\frac{A_{0}^{2} b_{0,0}+A_{0} A_{1} b_{0,1}+A_{1}^{2} b_{1,1}}{A_{0}^{5} B_{1}}, \\
& b_{1,1}^{\prime}=\frac{B_{1} b_{1,1}}{A_{0}^{5}}, \\
& b_{0,1}^{\prime}=\frac{A_{0} b_{0,1}+2 A_{1} b_{1,1}}{A_{0}^{5}},
\end{aligned}
$$




$$
\begin{aligned}
& b_{1,2}^{\prime}=\frac{1}{A_{0}^{4} B_{1}}\left(B_{1}^{2} b_{1,2}+\left(2 B_{1} B_{3}-B_{2}^{2}\right) b_{1,4}\right), \\
& b_{1,4}^{\prime}=\frac{B_{1}}{A_{0}^{2}} b_{1,4} .
\end{aligned}
$$

The set $C E\left(\mu_{6}\right)$ is represented as a union of the following subsets

$$
\begin{aligned}
U_{6}^{1} & =\left\{L(\alpha) \in C E\left(\mu_{6}\right) \mid b_{1,1} \neq 0, b_{1,4} \neq 0\right\} ; \\
U_{6}^{2} & =\left\{L(\alpha) \in C E\left(\mu_{6}\right) \mid b_{1,1} \neq 0, b_{1,4}=0, b_{1,2} \neq 0\right\} ; \\
U_{6}^{3} & =\left\{L(\alpha) \in C E\left(\mu_{6}\right) \mid b_{1,1} \neq 0, b_{1,4}=b_{1,2}=0, \Delta \neq 0\right\} ; \\
U_{6}^{4} & =\left\{L(\alpha) \in C E\left(\mu_{6}\right) \mid b_{1,1} \neq 0, b_{1,4}=b_{1,2}=\Delta=0\right\} ; \\
U_{6}^{5} & =\left\{L(\alpha) \in C E\left(\mu_{6}\right) \mid b_{1,1}=0, b_{0,1} \neq 0, b_{1,4} \neq 0\right\} ; \\
U_{6}^{6} & =\left\{L(\alpha) \in C E\left(\mu_{6}\right) \mid b_{1,1}=0, b_{0,1} \neq 0, b_{1,4}=0, b_{1,2} \neq 0\right\} ; \\
U_{6}^{7} & =\left\{L(\alpha) \in C E\left(\mu_{6}\right) \mid b_{1,1}=0, b_{0,1} \neq 0, b_{1,4}=b_{1,2}=0\right\} ; \\
U_{6}^{8} & =\left\{L(\alpha) \in C E\left(\mu_{6}\right) \mid b_{1,1}=b_{0,1}=0, b_{0,0} \neq 0, b_{1,4} \neq 0\right\} ; \\
U_{6}^{9} & =\left\{L(\alpha) \in C E\left(\mu_{6}\right) \mid b_{1,1}=b_{0,1}=0, b_{0,0} \neq 0, b_{1,4}=0, b_{1,2} \neq 0\right\} ; \\
U_{6}^{10} & =\left\{L(\alpha) \in C E\left(\mu_{6}\right) \mid b_{1,1}=b_{0,1}=0, b_{0,0} \neq 0, b_{1,4}=b_{1,2}=0\right\} ; \\
U_{6}^{11} & =\left\{L(\alpha) \in C E\left(\mu_{6}\right) \mid b_{1,1}=b_{0,1}=b_{0,0}=0, b_{1,4} \neq 0\right\} ; \\
U_{6}^{12} & =\left\{L(\alpha) \in C E\left(\mu_{6}\right) \mid b_{1,1}=b_{0,1}=b_{0,0}=b_{1,4}=0, b_{1,2} \neq 0\right\} ; \\
U_{6}^{13} & =\left\{L(\alpha) \in C E\left(\mu_{6}\right) \mid b_{1,1}=b_{0,1}=b_{0,0}=b_{1,4}=b_{1,2}=0\right\} .
\end{aligned}
$$

Proposition 3.14.

(1) Two algebras $L(\alpha)$ and $L\left(\alpha^{\prime}\right)$ from $U_{6}^{1}$ are isomorphic if and only if

$$
\left(\frac{b_{1,4}^{\prime}}{b_{1,1}^{\prime}}\right)^{8} \Delta^{\prime 3}=\left(\frac{b_{1,4}}{b_{1,1}}\right)^{8} \Delta^{3}
$$

(2) For any $\lambda$ from $\mathbb{C}$ there exists $L(\alpha) \in U_{6}^{1}$ such that $\left(b_{1,4} / b_{1,1}\right)^{8} \Delta^{3}=\lambda$.

The orbits in $U_{6}^{1}$ can be parameterized as $L(\lambda, 0,1,0,1), \lambda \in \mathbb{C}$. Proposition 3.15.

(1) Two algebras $L(\alpha)$ and $L\left(\alpha^{\prime}\right)$ from $U_{6}^{2}$ are isomorphic if and only if

$$
\left(\frac{b_{1,2}^{\prime}}{b_{1,1}^{\prime}}\right)^{8} \Delta^{\prime}=\left(\frac{b_{1,2}}{b_{1,1}}\right)^{8} \Delta \text {. }
$$

(2) For any $\lambda$ from $\mathbb{C}$ there exists $L(\alpha) \in U_{6}^{2}$ such that $\left(b_{1,2} / b_{1,1}\right)^{8} \Delta=\lambda$.

Then orbits in $U_{6}^{2}$ can be parameterized as $L(\lambda, 0,1,1,0), \lambda \in \mathbb{C}$. 
Proposition 3.16. The subsets $U_{6}^{3}, U_{6}^{4}, U_{6}^{5}, U_{6}^{6}, U_{6}^{7}, U_{6}^{8}, U_{6}^{9}, U_{6}^{10}, U_{6}^{11}, U_{6}^{12}$, and $U_{6}^{13}$ are single orbits with representatives

$$
\begin{gathered}
L(1,0,1,0,0), \quad L(0,0,1,0,0), \quad L(0,1,0,0,1), \quad L(0,1,0,1,0), \\
L(0,1,0,0,0), \quad L(1,0,0,0,1), \quad L(1,0,0,1,0), \quad L(1,0,0,0,0), \\
L(0,0,0,0,1), \quad L(0,0,0,1,0) \quad \text { and } L(0,0,0,0,0),
\end{gathered}
$$

respectively.

3.4. Isomorphism classes in $\boldsymbol{C E}\left(\boldsymbol{\mu}_{7}\right)$. From the interrelations between $b_{i, j}$ (see the multiplication table of $\left.C E\left(\mu_{n}\right)\right)$ we get $b_{2,5}=-b_{3,4}=b, b_{2,3}=-b_{1,4}$. Hence $L(\alpha)$, where $\alpha=\left(b_{0,0}, b_{0,1}, b_{1,1}, b_{1,2}, b_{1,4}, b\right)$, designates an element of $C E\left(\mu_{7}\right)$.

THEOREM 3.17 (Isomorphism criterion for $C E\left(\mu_{7}\right)$ ). Two filiform Leibniz algebras $L(\alpha)$ and $L\left(\alpha^{\prime}\right)$ from $C E\left(\mu_{7}\right)$ are isomorphic if and only if there exist $A_{0}, A_{1}, B_{i} \in \mathbb{C}$, $1 \leq i \leq 5$, such that $A_{0} B_{1}\left(A_{0}+A_{1} b\right) \neq 0$, and the following equalities hold:

$$
\begin{aligned}
b_{0,0}^{\prime} & =\frac{A_{0}^{2} b_{0,0}+A_{0} A_{1} b_{0,1}+A_{1}^{2} b_{1,1}}{A_{0}^{5} B_{1}\left(A_{0}+A_{1} b\right)}, \\
b_{0,1}^{\prime} & =\frac{A_{0} b_{0,1}+2 A_{1} b_{1,1}}{A_{0}^{5}\left(A_{0}+A_{1} b\right)}, \\
b_{1,1}^{\prime} & =\frac{B_{1} b_{1,1}}{A_{0}^{5}\left(A_{0}+A_{1} b\right)}, \\
b_{1,2}^{\prime} & =\frac{B_{1}^{2} b_{1,2}+\left(2 B_{1} B_{3}-B_{2}^{2}\right) b_{1,4}+\left(2 B_{2} B_{4}-2 B_{1} B_{5}-B_{3}^{3}\right) b}{2 A_{0}^{4} B_{1}\left(A_{0}+A_{1} b\right)}, \\
b_{1,4}^{\prime} & =-\frac{B_{1} b_{1,4}+\left(2 B_{1} B_{3}-B_{2}^{2}\right) b}{A_{0}^{2} B_{1}\left(A_{0}+A_{1} b\right)}, \\
b^{\prime} & =\frac{B b}{A_{0}+A_{1} b} .
\end{aligned}
$$

The class $C E\left(\mu_{7}\right)$ is represented as a union of the following subsets.

$$
\begin{aligned}
& U_{7}^{1}=\left\{L(\alpha) \in C E\left(\mu_{7}\right) \mid b \neq 0, b_{1,1} \neq 0\right\} ; \\
& U_{7}^{2}=\left\{L(\alpha) \in C E\left(\mu_{7}\right) \mid b \neq 0, b_{1,1}=0, b_{0,1} \neq 0\right\} ; \\
& U_{7}^{3}=\left\{L(\alpha) \in C E\left(\mu_{7}\right) \mid b \neq 0, b_{1,1}=b_{0,1}=0, b_{0,0} \neq 0\right\} ; \\
& U_{7}^{4}=\left\{L(\alpha) \in C E\left(\mu_{7}\right) \mid b \neq 0, b_{1,1}=b_{0,1}=b_{0,0}=0\right\} ; \\
& U_{7}^{5}=\left\{L(\alpha) \in C E\left(\mu_{7}\right) \mid b=0, b_{1,4} \neq 0, b_{1,1} \neq 0\right\} ; \\
& U_{7}^{6}=\left\{L(\alpha) \in C E\left(\mu_{7}\right) \mid b=0, b_{1,4} \neq 0, b_{1,1}=0, b_{0,1} \neq 0\right\} ; \\
& U_{7}^{7}=\left\{L(\alpha) \in C E\left(\mu_{7}\right) \mid b=0, b_{1,4} \neq 0, b_{1,1}=b_{0,1}=0, b_{0,0} \neq 0\right\} ; \\
& U_{7}^{8}=\left\{L(\alpha) \in C E\left(\mu_{7}\right) \mid b=0, b_{1,4} \neq 0, b_{1,1}=b_{0,1}=b_{0,0}=0\right\} ;
\end{aligned}
$$




$$
\begin{aligned}
& U_{7}^{9}=\left\{L(\alpha) \in C E\left(\mu_{7}\right) \mid b=b_{1,4}=0, b_{1,2} \neq 0, b_{1,1} \neq 0\right\} \\
& U_{7}^{10}=\left\{L(\alpha) \in C E\left(\mu_{7}\right) \mid b=b_{1,4}=0, b_{1,2} \neq 0, b_{1,1}=0, b_{0,1} \neq 0\right\} \\
& U_{7}^{11}=\left\{L(\alpha) \in C E\left(\mu_{7}\right) \mid b=b_{1,4}=0, b_{1,2} \neq 0, b_{1,1}=b_{0,1}=0, b_{0,0} \neq 0\right\} \\
& U_{7}^{12}=\left\{L(\alpha) \in C E\left(\mu_{7}\right) \mid b=b_{1,4}=0, b_{1,2} \neq 0, b_{1,1}=b_{0,1}=b_{0,0}=0\right\} \\
& U_{7}^{13}=\left\{L(\alpha) \in C E\left(\mu_{7}\right) \mid b=b_{1,4}=b_{1,2}=0, b_{1,1} \neq 0, \Delta \neq 0\right\} \\
& U_{7}^{14}=\left\{L(\alpha) \in C E\left(\mu_{7}\right) \mid b=b_{1,4}=b_{1,2}=0, b_{1,1} \neq 0, \Delta=0\right\} \\
& U_{7}^{15}=\left\{L(\alpha) \in C E\left(\mu_{7}\right) \mid b=b_{1,4}=b_{1,2}=b_{1,1}=0, b_{0,1} \neq 0\right\} \\
& U_{7}^{16}=\left\{L(\alpha) \in C E\left(\mu_{7}\right) \mid b=b_{1,4}=b_{1,2}=b_{1,1}=b_{0,1}=0, b_{0,0} \neq 0\right\} \\
& U_{7}^{17}=\left\{L(\alpha) \in C E\left(\mu_{7}\right) \mid b=b_{1,4}=b_{1,2}=b_{1,1}=b_{0,1}=b_{0,0}=0\right\} .
\end{aligned}
$$

\section{PROPOSITION 3.18.}

(1) Two algebras $L(\alpha)$ and $L\left(\alpha^{\prime}\right)$ from $U_{7}^{1}$ are isomorphic if and only if

$$
\left(\frac{b^{\prime}}{2 b_{1,1}^{\prime}-b_{0,1}^{\prime} b^{\prime}}\right)^{2} \Delta^{\prime}=\left(\frac{b}{2 b_{1,1}-b_{0,1} b}\right)^{2} \Delta .
$$

(2) For any $\lambda$ from $\mathbb{C}$ there exists $L(\alpha) \in U_{7}^{1}$ such that $\left(b /\left(2 b_{1,1}-b_{0,1} b\right)\right)^{2} \Delta=\lambda$.

Then orbits in $U_{7}^{1}$ can be parameterized as $L(\lambda, 0,1,0,0,1), \lambda \in \mathbb{C}$. PROPOSITION 3.19.

(1) Two algebras $L(\alpha)$ and $L\left(\alpha^{\prime}\right)$ from $U_{7}^{5}$ are isomorphic if and only if

$$
\left(\frac{b_{1,4}^{\prime}}{b_{1,1}^{\prime}}\right)^{10} \Delta^{\prime 3}=\left(\frac{b_{1,4}}{b_{1,1}}\right)^{10} \Delta^{3}
$$

(2) For any $\lambda$ from $\mathbb{C}$ there exists $L(\alpha) \in U_{7}^{5}$ such that $\left(b_{1,4} / b_{1,1}\right)^{10} \Delta^{3}=\lambda$.

Then orbits in $U_{7}^{5}$ can be parameterized as $L(\lambda, 0,1,0,1,0), \lambda \in \mathbb{C}$.

PROPOSITION 3.20.

(1) Two algebras $L(\alpha)$ and $L\left(\alpha^{\prime}\right)$ from $U_{7}^{9}$ are isomorphic if and only if

$$
\left(\frac{b_{1,2}^{\prime}}{b_{1,1}^{\prime}}\right)^{10} \Delta^{\prime 3}=\left(\frac{b_{1,2}}{b_{1,1}}\right)^{10} \Delta^{3}
$$

(2) For any $\lambda$ from $\mathbb{C}$ there exists $L(\alpha) \in U_{7}^{9}$ such that $\left(b_{1,2} / b_{1,1}\right)^{10} \Delta^{3}=\lambda$.

Then orbits in $U_{7}^{9}$ can be parameterized as $L(\lambda, 0,1,1,0,0), \lambda \in \mathbb{C}$. 
Proposition 3.21. The subsets $U_{7}^{2}, U_{7}^{3}, U_{7}^{4}, U_{7}^{6}, U_{7}^{7}, U_{7}^{8}, U_{7}^{10}, U_{7}^{11}, U_{7}^{12}, U_{7}^{13}, U_{7}^{14}$, $U_{7}^{15}, U_{7}^{16}$, and $U_{7}^{17}$ are single orbits with representatives

$L(0,1,0,0,0,1), \quad L(1,0,0,0,0,1), \quad L(0,0,0,0,0,1), \quad L(0,1,0,0,1,0)$, $L(1,0,0,0,1,0), \quad L(0,0,0,0,1,0), \quad L(0,1,0,1,0,0), \quad L(1,0,0,1,0,0)$, $L(0,0,0,1,0,0), \quad L(1,0,1,0,0,0), \quad L(0,0,1,0,0,0), \quad L(0,1,0,0,0,0)$, $L(1,0,0,0,0,0)$ and $L(0,0,0,0,0,0)$,

respectively.

3.5. Isomorphism classes in $\boldsymbol{C E}\left(\boldsymbol{\mu}_{\mathbf{8}}\right)$. It is easy to see that $b_{1,4}=-b_{3,2}, b_{1,6}=$ $b_{3,4}=b_{5,2}$ and $b=0$. An element of $C E\left(\mu_{8}\right)$ is denoted by $L(\alpha)$, where $\alpha=$ $\left(b_{0,0}, b_{0,1}, b_{1,1}, b_{1,2}, b_{1,4}, b_{1,6}\right)$.

THEOREM 3.22 (Isomorphism criterion for $C E\left(\mu_{8}\right)$ ). Two filiform Leibniz algebras $L(\alpha)$ and $L\left(\alpha^{\prime}\right)$ from $C E\left(\mu_{8}\right)$ are isomorphic if and only if there exist $A_{0}, A_{1}, B_{i} \in \mathbb{C}$, $1 \leq i \leq 5$, such that $A_{0} B_{1} \neq 0$ and the following equalities hold:

$$
\begin{aligned}
& b_{0,0}^{\prime}=\frac{A_{0}^{2} b_{0,0}+A_{0} A_{1} b_{0,1}+A_{1}^{2} b_{1,1}}{A_{0}^{7} B_{1}}, \\
& b_{0,1}^{\prime}=\frac{2 A_{1} b_{1,1}+A_{0} b_{0,1}}{A_{0}^{7}}, \\
& b_{1,1}^{\prime}=\frac{B_{1} b_{1,1}}{A_{0}^{7}}, \\
& b_{1,2}^{\prime}=\frac{B_{1}^{2} b_{1,2}+\left(2 B_{1} B_{3}-B_{2}^{2}\right) b_{1,4}+\left(2 B_{1} B_{5}-2 B_{2} B_{4}+B_{3}^{2}\right) b_{1,6}}{A_{0}^{6} B_{1}}, \\
& b_{1,4}^{\prime}=\frac{B_{1}^{2} b_{1,4}+\left(2 B_{1} B_{3}-B_{2}^{2}\right) b_{1,6}}{A_{0}^{4} B_{1}}, \\
& b_{1,6}^{\prime}=\frac{B_{1} b_{1,6}}{A_{0}^{2}} .
\end{aligned}
$$

The class $C E\left(\mu_{8}\right)$ is represented as a union of the following subsets.

$$
\begin{aligned}
U_{8}^{1} & =\left\{L(\alpha) \in C E\left(\mu_{8}\right) \mid b_{1,6} \neq 0, b_{1,1} \neq 0\right\} ; \\
U_{8}^{2} & =\left\{L(\alpha) \in C E\left(\mu_{8}\right) \mid b_{1,6} \neq 0, b_{1,1}=0, b_{0,1} \neq 0\right\} ; \\
U_{8}^{3} & =\left\{L(\alpha) \in C E\left(\mu_{8}\right) \mid b_{1,6} \neq 0, b_{1,1}=b_{0,1}=0, b_{0,0} \neq 0\right\} ; \\
U_{8}^{4} & =\left\{L(\alpha) \in C E\left(\mu_{8}\right) \mid b_{1,6} \neq 0, b_{1,1}=b_{0,1}=b_{0,0}=0\right\} ; \\
U_{8}^{5} & =\left\{L(\alpha) \in C E\left(\mu_{8}\right) \mid b_{1,6}=0, b_{1,4} \neq 0, b_{1,1} \neq 0\right\} ; \\
U_{8}^{6} & =\left\{L(\alpha) \in C E\left(\mu_{8}\right) \mid b_{1,6}=0, b_{1,4} \neq 0, b_{1,1}=0, b_{0,1} \neq 0\right\} ; \\
U_{8}^{7} & =\left\{L(\alpha) \in C E\left(\mu_{8}\right) \mid b_{1,6}=0, b_{1,4} \neq 0, b_{1,1}=b_{0,1}=0, b_{0,0} \neq 0\right\} ;
\end{aligned}
$$




$$
\begin{aligned}
U_{8}^{8} & =\left\{L(\alpha) \in C E\left(\mu_{8}\right) \mid b_{1,6}=0, b_{1,4} \neq 0, b_{1,1}=b_{0,1}=b_{0,0}=0\right\} ; \\
U_{8}^{9} & =\left\{L(\alpha) \in C E\left(\mu_{8}\right) \mid b_{1,6}=b_{1,4}=0, b_{1,2} \neq 0, b_{1,1} \neq 0\right\} ; \\
U_{8}^{10} & =\left\{L(\alpha) \in C E\left(\mu_{8}\right) \mid b_{1,6}=b_{1,4}=0, b_{1,2} \neq 0, b_{1,1}=0, b_{0,1} \neq 0\right\} ; \\
U_{8}^{11} & =\left\{L(\alpha) \in C E\left(\mu_{8}\right) \mid b_{1,6}=b_{1,4}=0, b_{1,2} \neq 0, b_{1,1}=b_{0,1}=0, b_{0,0} \neq 0\right\} ; \\
U_{8}^{12} & =\left\{L(\alpha) \in C E\left(\mu_{8}\right) \mid b_{1,6}=b_{1,4}=0, b_{1,2} \neq 0, b_{1,1}=b_{0,1}=b_{0,0}=0\right\} ; \\
U_{8}^{13} & =\left\{L(\alpha) \in C E\left(\mu_{8}\right) \mid b_{1,6}=b_{1,4}=b_{1,2}=0, b_{1,1} \neq 0, \Delta \neq 0\right\} ; \\
U_{8}^{14} & =\left\{L(\alpha) \in C E\left(\mu_{8}\right) \mid b_{1,6}=b_{1,4}=b_{1,2}=0, b_{1,1} \neq 0, \Delta=0\right\} ; \\
U_{8}^{15} & =\left\{L(\alpha) \in C E\left(\mu_{8}\right) \mid b_{1,6}=b_{1,4}=b_{1,2}=b_{1,1}=0, b_{0,1} \neq 0\right\} ; \\
U_{8}^{16} & =\left\{L(\alpha) \in C E\left(\mu_{8}\right) \mid b_{1,6}=b_{1,4}=b_{1,2}=b_{1,1}=b_{0,1}=0, b_{0,0} \neq 0\right\} ; \\
U_{8}^{17} & =\left\{L(\alpha) \in C E\left(\mu_{8}\right) \mid b_{1,6}=b_{1,4}=b_{1,2}=b_{1,1}=b_{0,1}=b_{0,0}=0\right\} .
\end{aligned}
$$

The following propositions describe $U_{8}^{1}, U_{8}^{5}$ and $U_{8}^{9}$.

PROPOSITION 3.23.

(1) Two algebras $L(\alpha)$ and $L\left(\alpha^{\prime}\right)$ from $U_{8}^{1}$ are isomorphic if and only if

$$
\left(\frac{b_{1,6}^{\prime}}{b_{1,1}^{\prime}}\right)^{12} \Delta^{\prime 5}=\left(\frac{b_{1,6}}{b_{1,1}}\right)^{12} \Delta^{5}
$$

(2) For any $\lambda$ from $\mathbb{C}$ there exists $L(\alpha) \in U_{8}^{1}$ such that $\left(b_{1,6} / b_{1,1}\right)^{12} \Delta^{5}=\lambda$.

Orbits in $U_{8}^{1}$ can be parameterized as $L(\lambda, 0,1,0,0,1), \lambda \in \mathbb{C}$.

PROPOSITION 3.24.

(1) Two algebras $L(\alpha)$ and $L\left(\alpha^{\prime}\right)$ from $U_{8}^{5}$ are isomorphic if and only if

$$
\left(\frac{b_{1,1}^{\prime}}{b_{1,4}^{\prime}}\right)^{4} \frac{1}{\Delta^{\prime}}=\left(\frac{b_{1,1}}{b_{1,4}}\right)^{4} \frac{1}{\Delta} \text {. }
$$

(2) For any $\lambda$ from $\mathbb{C}$ there exists $L(\alpha) \in U_{8}^{5}$ such that $\left(b_{1,1} / b_{1,4}\right)^{4}(1 / \Delta)=\lambda$.

The parametrization of orbits in $U_{8}^{5}$ can be viewed as $L(\lambda, 0,1,0,1,0), \lambda \in \mathbb{C}$.

PROPOSITION 3.25.

(1) Two algebras $L(\alpha)$ and $L\left(\alpha^{\prime}\right)$ from $U_{8}^{9}$ are isomorphic if and only if

$$
\left(\frac{b_{1,2}^{\prime}}{b_{1,1}^{\prime}}\right)^{12} \Delta^{\prime}=\left(\frac{b_{1,2}}{b_{1,1}}\right)^{12} \Delta .
$$

(2) For any $\lambda \in \mathbb{C}$ there exists $L(\alpha) \in U_{8}^{9}$ such that $\left(b_{1,2} / b_{1,1}\right)^{12} \Delta=\lambda$.

The set of orbits in $U_{8}^{9}$ can be parameterized as $L(\lambda, 0,1,1,0,0), \lambda \in \mathbb{C}$. 
Proposition 3.26. The subsets $U_{8}^{2}, U_{8}^{3}, U_{8}^{4}, U_{8}^{6}, U_{8}^{7}, U_{8}^{8}, U_{8}^{10}, U_{8}^{11}, U_{8}^{12}, U_{8}^{13}, U_{8}^{14}$, $U_{8}^{15}, U_{8}^{16}$, and $U_{8}^{17}$ are single orbits with representatives

$L(0,1,0,0,0,1), \quad L(1,0,0,0,0,1), \quad L(0,0,0,0,0,1), \quad L(0,1,0,0,1,0)$, $L(1,0,0,0,1,0), \quad L(0,0,0,0,1,0), \quad L(0,1,0,1,0,0), \quad L(1,0,0,1,0,0)$, $L(0,0,0,1,0,0), \quad L(1,0,1,0,0,0), \quad L(0,0,1,0,0,0), \quad L(0,1,0,0,0,0)$, $L(1,0,0,0,0,0)$ and $L(0,0,0,0,0,0)$,

respectively.

\section{Acknowledgements}

We are grateful to Professor B. A. Omirov for helpful discussions. The authors also would like to thank the referee and Professor D. E. Taylor for constructive and valuable comments that have substantially improved the presentation of the paper.

\section{References}

[1] S. Albeverio, B. A. Omirov and I. S. Rakhimov, 'Classification of four-dimensional nilpotent complex Leibniz algebras', Extracta Math. 21(3) (2006), 197-210.

[2] J. R. Gomez, A. Jimenez-Merchan and Y. Khakimdjanov, 'Low-dimensional filiform Lie algebras', J. Pure Appl. Algebra 130 (1998), 133-158.

[3] You. B. Hakimjanov (Khakimdjanov), 'Variété des lois d'algèbres de Lie nilpotentes', Geom. Dedicata 40 (1991), 229-295.

[4] J.-L. Loday, 'Une version noncommutative des algèbres de Lie: les algèbres de Leibniz', Enseign. Math. 39 (1993), 269-293.

[5] J.-L. Loday and T. Pirashvili, 'Universal enveloping algebras of Leibniz algebras and (co)homology', Math. Ann. 296 (1993), 139-158.

[6] B. A. Omirov and I. S. Rakhimov, 'On Lie-like complex filiform Leibniz algebras', Bull. Aust. Math. Soc. 79 (2009), 391-404.

[7] K. A. Umlauf, Über die Zusammensetzung der endlichen continuierlichen Transformationsgruppen, insbesondere der Gruppen vom Range Null, Inaugural-Dissertation, Universität Leipzig (Von Breitkopf \& Härtel, Leipzig, 1891).

ISAMIDDIN S. RAKHIMOV, Institute for Mathematical Research (INSPEM)

and

Department of Mathematics, FS, Universiti Putra Malaysia, UPM, 43400, Serdang, Selangor Darul Ehsan, Malaysia

e-mail: risamiddin@gmail.com

MUNTHER A. HASSAN, Institute for Mathematical Research (INSPEM), Serdang, Selangor Darul Ehsan, Malaysia

e-mail: munther_abd@yahoo.com 